\title{
Existence and Global Exponential Stability of Almost Periodic Solutions for a Class of Delay Duffing Equations on Time Scales
}

\author{
Li Yang and Yongkun Li \\ Department of Mathematics, Yunnan University, Kunming, Yunnan 650091, China \\ Correspondence should be addressed to Yongkun Li; yklie@ynu.edu.cn
}

Received 19 March 2014; Revised 31 May 2014; Accepted 4 June 2014; Published 16 June 2014

Academic Editor: Chuanzhi Bai

Copyright (C) 2014 L. Yang and Y. Li. This is an open access article distributed under the Creative Commons Attribution License, which permits unrestricted use, distribution, and reproduction in any medium, provided the original work is properly cited.

Based on the exponential dichotomy of linear dynamic equations on time scales, we obtain some sufficient conditions for the existence and global exponential stability of almost periodic solutions for a class of Duffing equations with time-varying delays on time scales. We also present numerical examples to show the feasibility of obtained results. The results of this paper are completely new even when the time scale $\mathbb{T}=\mathbb{R}$ or $\mathbb{Z}$ and are complementary to the previously known results.

\section{Introduction}

As we know Duffing equations describe the motion of a mechanical system in a twin-well potential field. Due to their promising potential applications in areas of physics, mechanics, and engineering technique fields, various kinds of dynamic behaviors of Duffing equations have been studied by many authors (see [1-4]). Among them, the existence of almost periodic solutions is an important topic. For example, authors of [5] investigated the following Duffing equation:

$$
x^{\prime \prime}(t)-x(t)+x^{m}(t-\tau)=p(t), \quad m>1,
$$

where $p(t)$ is an almost periodic function on $\mathbb{R}, m>1$ is an integer, and $\tau>0$ is a constant. Under the assumption that

$(S)$

$$
\sup _{t \in R}|p(t)| \leq\left(\frac{1}{m}\right)^{1 /(m-1)}\left(1-\frac{1}{m}\right)
$$

by using the exponential dichotomy and some mathematical analysis technique; they obtained some criteria for the existence of almost periodic solutions for (1); in [6], authors investigated the following Duffing equation:

$$
x^{\prime \prime}(t)+c x^{\prime}(t)-a x(t)+b x^{m}(t-\tau(t))=p(t), \quad m>1 .
$$

By using differential inequalities and some mathematical analysis technique, they obtained some criteria for the existence of almost periodic solutions for (3) without assuming condition $(S)$. After that, authors of [7] considered the existence of almost periodic solutions for the following Duffing equation:

$$
x^{\prime \prime}(t)+c x^{\prime}(t)-a x(t)+b x^{m}(t-\tau(t))=p(t), \quad m>1 .
$$

Taking

$$
\begin{gathered}
y=x^{\prime}+\xi x-Q_{1}(t), \\
Q_{2}(t)=p(t)+(\xi-c) Q_{1}(t)-Q_{1}^{\prime}(t),
\end{gathered}
$$

(4) is transformed into the following system:

$$
\begin{gathered}
x^{\prime}(t)=-\xi x(t)+y(t)+Q_{1}(t), \\
y^{\prime}(t)=-(c-\xi) y(t)+(a-\xi(\xi-c)) x(t) \\
-b x^{m}(t-\tau(t))+Q_{2}(t) .
\end{gathered}
$$

Then, considering the coefficients in (4) are usually time-varying, authors of [8] gave conditions to guarantee 
the existence of almost periodic solutions of the following equation:

$$
\begin{gathered}
x^{\prime}(t)=-\delta_{1}(t) x(t)+y(t)+Q_{1}(t), \\
y^{\prime}(t)=\delta_{2}(t) y(t)+\left[\alpha(t)-\delta_{2}^{2}(t)\right] x(t) \\
-\beta(t) x^{m}(t-\tau(t))+Q_{2}(t) .
\end{gathered}
$$

However, there have been few results about the discrete analogue of the above systems. In fact, both continuous and discrete systems are very important in implementation and applications. But it is troublesome to study the dynamics for continuous and discrete systems, respectively. Therefore, it is significant to study that on time scales which can unify the continuous and discrete situations.

Motivated by the above, in this paper, we study the almost periodic solutions of the following Duffing equation on time scale $\mathbb{T}$ :

$$
\begin{aligned}
& \left(x^{\Delta}\right)^{\Delta}(t)+c(t) x^{\Delta}(t)-a(t) x(t)+b(t) x^{m}(t-\tau(t)) \\
& \quad=p(t),
\end{aligned}
$$

where $t \in \mathbb{T}, t-\tau(t) \in \mathbb{T}, \mathbb{T}$ is an almost periodic time scale, and $m>1$ is a constant.

Remark 1. If $\mathbb{T}=\mathbb{R}, c(t) \equiv 0$ and $b(t) \equiv b$, then (8) reduces to (3). If $\mathbb{T}=\mathbb{R}, c(t) \equiv c, a(t) \equiv a$, and $b(t) \equiv b$, then (8) reduces to (4), which can be transformed to (7). Hence, (3), (4), and (7) are all special cases of (8).

Let $\delta_{1}(t)$ be a continuous function with continuous $\Delta$ derivatives on $\mathbb{T}$. Set

$$
y(t)=x^{\Delta}(t)+\delta_{1}(t) x(t)
$$

then we transform (8) into the following system:

$$
\begin{gathered}
x^{\Delta}(t)=-\delta_{1}(t) x(t)+y(t), \\
y^{\Delta}(t)=-\delta_{2}(t) y(t)+\beta(t) x(t)-b(t) x^{m}(t-\tau(t))+p(t),
\end{gathered}
$$

where $\delta_{2}(t)=c(t)-\delta_{1}(\sigma(t)), \beta(t)=a(t)+\delta_{1}^{\Delta}(t)+\delta_{1}(t) \delta_{2}(t)$.

To the best of our knowledge, up to now, there are no results available on the existence and global exponential stability of almost periodic solution for Duffing equations on time scales. Our main aim of this paper is to study the existence of almost periodic solutions for (10) with the exponential dichotomy of linear dynamic equations on time scales. Moreover, we also study the global exponential stability of almost periodic solutions for (10), which was not considered in [5-8]. Our results of this paper are new and complementary to the previously known results.

For convenience, we denote $[a, b]_{\mathbb{T}}=\{t \mid t \in[a, b] \cap$ $\mathbb{T}$ \}. For an almost periodic function $f: \mathbb{T} \rightarrow \mathbb{R}$, denote $f^{+}=\sup _{t \in \mathbb{T}}|f(t)|, f^{-}=\inf _{t \in \mathbb{T}}|f(t)|$. Set $\mathbb{X}=\{\varphi=$ $\left.\left(\varphi_{1}(t), \varphi_{2}(t)\right)^{T}\right) \mid \varphi_{i}(t) \in C(\mathbb{T}, \mathbb{R}) ; \varphi_{i}$ is an almost periodic function on $\mathbb{T}, i=1,2\}$, with the norm $\|\varphi\|=$ $\max \left\{\sup _{t \in \mathbb{I}}\left|\varphi_{1}(t)\right| \sup _{t \in \mathbb{T}}\left|\varphi_{2}(t)\right|\right\}$; then $\mathbb{X}$ is a Banach space. The initial condition of (10) is

$$
x(s)=\varphi_{1}(s), \quad y(s)=\varphi_{2}(s), \quad s \in\left[-\tau^{+}, 0\right]_{\mathbb{T}},
$$

where $\varphi_{i} \in C\left(\left[-\tau^{+}, 0\right]_{\mathbb{T}}, \mathbb{R}\right)$.

Throughout this paper, we assume that the following condition holds:

$\left(H_{1}\right) \beta(t), b(t), p(t) \in C(\mathbb{T}, \mathbb{R}), \delta_{1}(t), \delta_{2}(t) \in C\left(\mathbb{T}, \mathbb{R}^{+}\right)$ with $-\delta_{1},-\delta_{2} \in \mathscr{R}^{+}$are all almost periodic functions, where $\mathscr{R}^{+}$denotes the set of positively regressive functions from $\mathbb{T}$ to $\mathbb{R}$, and $t-\tau(t) \in C\left(\mathbb{T}, \mathbb{T} \cap \mathbb{R}^{+}\right)$is almost periodic, where $\tau(t) \in C\left(\mathbb{T}, \mathbb{R}^{+}\right)$.

This paper is organized as follows. In Section 2, we introduce some notations and definitions and state some preliminary results which are needed in later sections. In Section 3, we establish some sufficient conditions for the existence of almost periodic solutions of (10) and prove that these conditions also guarantee the global exponential stability of almost periodic solutions of (10). In Section 4, we give examples to illustrate the feasibility of our results obtained in previous sections. We draw a conclusion in Section 5.

\section{Preliminaries}

In this section, we introduce some definitions and state some preliminary results.

Definition 2 (see [9]). Let $\mathbb{T}$ be a nonempty closed subset (time scale) of $\mathbb{R}$. The forward and backward jump operators $\sigma, \rho: \mathbb{T} \rightarrow \mathbb{T}$ and the graininess $\mu: \mathbb{T} \rightarrow \mathbb{R}_{+}$are defined, respectively, by

$$
\sigma(t)=\inf \{s \in \mathbb{T}: s>t\}, \quad \rho(t)=\sup \{s \in \mathbb{T}: s<t\},
$$

$$
\mu(t)=\sigma(t)-t .
$$

Lemma 3 (see [9]). Assume that $p, q: \mathbb{T} \rightarrow \mathbb{R}$ are two regressive functions; then
(i) $e_{0}(t, s) \equiv 1$ and $e_{p}(t, t) \equiv 1$;
(ii) $e_{p}(\sigma(t), s)=(1+\mu(t) p(t)) e_{p}(t, s)$;
(iii) $e_{p}(t, s)=1 / e_{p}(s, t)=e_{\ominus p}(s, t)$;
(iv) $e_{p}(t, s) e_{p}(s, r)=e_{p}(t, r)$;
(v) $e_{p}(t, s)^{\Delta}=(p)(t) e_{p}(t, s)$.

Lemma 4 (see [9]). Let $f, g$ be $\Delta$-differentiable functions on $\mathbb{T}$; then

(i) $\left(v_{1} f+v_{2} g\right)^{\Delta}=v_{1} f^{\Delta}+v_{2} g^{\Delta}$, for any constants $v_{1}, v_{2}$;

(ii) $(f g)^{\Delta}(t)=f^{\Delta}(t) g(t)+f(\sigma(t)) g^{\Delta}(t)=f(t) g^{\Delta}(t)+$ $f^{\Delta}(t) g(\sigma(t))$

(iii) if $f^{\Delta} \geq 0$, then $f$ is nondecreasing. 
Lemma 5 (see [9]). Assume that $p(t) \geq 0$ for $t \geq s$; then $e_{p}(t, s) \geq 1$.

Definition 6 (see [9]). A function $f: \mathbb{T} \rightarrow \mathbb{R}$ is called regressive if $1+\mu(t) f(t) \neq 0$ for all $t \in \mathbb{T}$. The set of all regressive functions $f: \mathbb{T} \rightarrow \mathbb{R}$ will be denoted by $\mathscr{R}$. A function $f: \mathbb{T} \rightarrow \mathbb{R}$ is positively regressive if $1+\mu(t) f(t)>0$ for all $t \in \mathbb{T}$. The set of all positively regressive functions $f: \mathbb{T} \rightarrow \mathbb{R}$ will be denoted by $\mathscr{R}^{+}$.

Lemma 7 (see [9]). Suppose that $p \in \mathscr{R}^{+}$; then

(i) $e_{p}(t, s)>0$, for all $t, s \in \mathbb{T}$;

(ii) if $p(t) \leq q(t)$ for all $t \geq s, t, s \in \mathbb{T}$, then $e_{p}(t, s) \leq$ $e_{q}(t, s)$ for all $t \geq s$.

Lemma 8 (see [9]). If $p \in \mathscr{R}$ and $a, b, c \in \mathbb{T}$, then

$$
\begin{gathered}
{\left[e_{p}(c, \cdot)\right]^{\Delta}=-p\left[e_{p}(c, \cdot)\right]^{\sigma},} \\
\int_{a}^{b} p(t) e_{p}(c, \sigma(t)) \Delta t=e_{p}(c, a)-e_{p}(c, b) .
\end{gathered}
$$

Definition 9 (see [10]). A time scale $\mathbb{T}$ is called an almost periodic time scale if

$$
\Pi:=\{\tau \in \mathbb{R}: t \pm \tau \in \mathbb{T}, \forall t \in \mathbb{T}\} \neq\{0\} .
$$

Definition 10 (see [10]). Let $\mathbb{T}$ be an almost periodic time scale. A function $f: \mathbb{T} \rightarrow \mathbb{R}^{n}$ is said to be almost periodic on $\mathbb{T}$, if, for any $\varepsilon>0$, the set

$$
E(\varepsilon, f)=\{\tau \in \Pi:|f(t+\tau)-f(t)|<\varepsilon, \forall t \in \mathbb{T}\}
$$

is relatively dense in $\mathbb{T}$; that is, for any $\varepsilon>0$, there exists a constant $l(\varepsilon)>0$ such that each interval of length $l(\varepsilon)$ contains at least one $\tau \in E(\varepsilon, f)$ such that

$$
|f(t+\tau)-f(t)|<\varepsilon, \quad \forall t \in \mathbb{T} .
$$

The set $E(\varepsilon, f)$ is called the $\varepsilon$-translation set of $f(t), \tau$ is called the $\varepsilon$-translation number of $f(t)$, and $l(\varepsilon)$ is called the inclusion of $E(\varepsilon, f)$.

Lemma 11 (see [10]). If $f \in C\left(\mathbb{T}, \mathbb{R}^{n}\right)$ is an almost periodic function, then $f$ is bounded on $\mathbb{T}$.

Lemma 12 (see [10]). If $f, g \in C\left(\mathbb{T}, \mathbb{R}^{n}\right)$ are almost periodic functions, then $f+g, f g$ are also almost periodic.

Lemma 13 (see [10]). If $f \in C\left(\mathbb{T}, \mathbb{R}^{n}\right)$ is almost periodic and $F(\cdot)$ is uniformly continuous on the value field of $f$, then $F \circ f$ is almost periodic.

Definition 14 (see [11]). Let $x \in \mathbb{R}^{n}$ and $A(t)$ be a $n \times n$ matrixvalued function on $\mathbb{T}$; the linear system

$$
x^{\Delta}(t)=A(t) x(t), \quad t \in \mathbb{T}
$$

is said to admit an exponential dichotomy on $\mathbb{T}$ if there exist positive constants $k_{i}, \alpha_{i}, i=1,2$, projection $P$ and the fundamental solution matrix $X(t)$ of (17) satisfying

$$
\begin{gathered}
\left|X(t) P X^{-1}(s)\right| \leq k_{1} e_{\ominus \alpha_{1}}(t, s), \quad s, t \in \mathbb{T}, t \geq s, \\
\left|X(t)(I-P) X^{-1}(s)\right| \leq k_{2} e_{\ominus \alpha_{2}}(s, t), \quad s, t \in \mathbb{T}, t \leq s,
\end{gathered}
$$

where $|\cdot|$ is a matrix norm on $\mathbb{T}$; that is, if $A=\left(a_{i j}\right)_{n \times m}$, then we can take $|A|=\left(\sum_{i=1}^{n} \sum_{j=1}^{m}\left|a_{i j}\right|^{2}\right)^{1 / 2}$

Lemma 15 (see [10]). If (17) admits an exponential dichotomy, then the following almost periodic system

$$
x^{\Delta}(t)=A(t) x(t)+g(t), \quad t \in \mathbb{T}
$$

has an almost periodic solution as follows:

$$
\begin{aligned}
x(t)= & \int_{-\infty}^{t} X(t) P X^{-1}(\sigma(s)) g(s) \Delta s \\
& -\int_{t}^{+\infty} X(t)(I-P) X^{-1}(\sigma(s)) g(s) \Delta s,
\end{aligned}
$$

where $X(t)$ is the fundamental solution matrix of (17).

Lemma 16 (see [11]). If $A(t)$ is a uniformly bounded $r d$ continuous $n \times n$ matrix-valued function on $\mathbb{T}$ and there is a $\delta>0$ such that

$$
\begin{array}{r}
\left|a_{i i}(t)\right|-\sum_{j \neq i}\left|a_{i j}(t)\right|-\frac{1}{2} \mu(t)\left(\sum_{j=1}^{n}\left|a_{i j}(t)\right|\right)^{2}-\delta^{2} \mu(t) \geq 2 \delta, \\
t \in \mathbb{T}, \quad i=1,2, \ldots, n,
\end{array}
$$

then (17) admits an exponential dichotomy on $\mathbb{T}$.

Definition 17. Let $z_{*}(t)=\left(x_{*}(t), y_{*}(t)\right)^{T}$ be an almost periodic solution of (10) with initial value $\varphi_{*}(s)=$ $\left(\varphi_{* 1}(s), \varphi_{* 2}(s)\right)^{T}$. If there exist positive constants $\lambda$ with $-\lambda \epsilon$ $\mathscr{R}^{+}$and $M>1$ such that for an arbitrary solution $z(t)=$ $(x(t), y(t))^{T}$ of $(8)$ with initial value $\varphi(s)=\left(\varphi_{1}(s), \varphi_{2}(s)\right)^{T}$ satisfies

$$
\begin{array}{r}
\left|z(t)-z_{*}(t)\right|_{1} \leq M\left\|\varphi-\varphi_{*}\right\| e_{-\lambda}\left(t, t_{0}\right), \\
t, t_{0} \in\left[-\tau^{+}, \infty\right)_{\mathbb{T}}, \quad t \geq t_{0},
\end{array}
$$

where $|z(t)|_{1}=\max \left\{\left|x(t)-x_{*}(t)\right|,\left|y(t)-y_{*}(t)\right|\right\}, \| \varphi-$ $\left.\varphi_{*} \|=\max \left\{\left|\varphi_{1}-\varphi_{* 1}\right|_{0},\left|\varphi_{2}-\varphi_{* 2}\right|_{0}\right\}\right\}$, and $\left|\varphi_{i}-\varphi_{* i}\right|_{0}=$ $\sup _{s \in\left[-\tau^{+}, 0\right]_{\mathbb{T}}}\left|\varphi_{1}(s)-\varphi_{* 1}(s)\right|, i=1,2$. Then the solution $z_{*}(t)$ is said to be globally exponentially stable.

\section{Main Results}

In this section, we will state and prove the existence and global exponential stability of almost periodic solutions of (10).

Let $\varphi^{0}(t)=\left(0, \varphi_{2}^{0}(t)\right)^{T}$, where $\varphi_{2}^{0}(t)=\int_{-\infty}^{t} e_{-\delta_{2}}(t$, $\sigma(s)) p(s) \Delta s$. Then $\left\|\varphi^{0}\right\| \leq L$, where $L=p^{+} / \delta_{2}^{-}$. We have the following theorem. 
Theorem 18. Let $\left(H_{1}\right)$ hold. Suppose further that

$\left(\mathrm{H}_{2}\right)$ there exists a positive constant $\delta$ such that

$\delta_{i}(t)-\frac{1}{2} \mu(t) \delta_{i}^{2}(t)-\delta^{2} \mu(t) \geq 2 \delta, \quad t \in \mathbb{T}, \quad i=1,2 ;$

$\left(\mathrm{H}_{3}\right)$ the following inequalities hold:

$$
\begin{gathered}
\theta=\max \left\{\frac{1}{\delta_{1}^{-}}, \frac{\beta^{+}+b^{+}}{\delta_{2}^{-}}\right\}<1, \\
\gamma=\max \left\{\frac{1}{\delta_{1}^{-}}, \frac{\beta^{+}+m b^{+}(2 L /(1-\theta))^{m-1}}{\delta_{2}^{-}}\right\}<1, \\
\frac{L}{1-\theta}<1 .
\end{gathered}
$$

Then (10) has a unique almost periodic solution in $\mathbb{X}_{0}=\{\varphi \in$ $\left.\mathbb{X} \mid\left\|\varphi-\varphi^{0}\right\| \leq \theta L /(1-\theta)\right\}$.

Proof. For any given $\varphi \in \mathbb{X}$, we consider the following almost periodic system:

$$
\begin{gathered}
x^{\Delta}(t)=-\delta_{1}(t) x(t)+\varphi_{2}(t), \\
y^{\Delta}(t)=-\delta_{2}(t) y(t)+\beta(t) \varphi_{1}(t) \\
-b(t) \varphi_{1}^{m}(t-\tau(t))+p(t) .
\end{gathered}
$$

Since $\left(\mathrm{H}_{2}\right)$ holds, it follows from Lemma 16 that the linear system

$$
\begin{aligned}
& x^{\Delta}(t)=-\delta_{1}(t) x(t), \\
& y^{\Delta}(t)=-\delta_{2}(t) y(t)
\end{aligned}
$$

admits an exponential dichotomy on $\mathbb{T}$. Thus, by Lemma 15 , we obtain that (25) has an almost periodic solution, which is expressed as follows:

$$
\begin{gathered}
x^{\varphi}(t)=\int_{-\infty}^{t} e_{-\delta_{1}}(t, \sigma(s)) \varphi_{2}(s) \Delta s, \\
y^{\varphi}(t)=\int_{-\infty}^{t} e_{-\delta_{2}}(t, \sigma(s)) \\
\times\left(\beta(s) \varphi_{1}(s)-b(s) \varphi_{1}^{m}(s-\tau(s))+p(s)\right) \Delta s .
\end{gathered}
$$

For every $\varphi \in \mathbb{X}_{0}$, we have $\|\varphi\| \leq\left\|\varphi-\varphi^{0}\right\|+\left\|\varphi^{0}\right\| \leq$ $(\theta L /(1-\theta))+L=L /(1-\theta)<1$. Define the following nonlinear operator:

$$
\Phi: \mathbb{X}_{0} \longrightarrow \mathbb{X}_{0}, \quad \varphi=\left(\varphi_{1}, \varphi_{2}\right)^{T} \longrightarrow z^{\varphi}=\left(x^{\varphi}, y^{\varphi}\right)^{T}
$$

We will show that $\Phi$ is a contraction.
At first, we show that for any $\varphi \in \mathbb{X}_{0}$, we have $\Phi \varphi \in \mathbb{X}_{0}$. Note that

$$
\begin{aligned}
& \left\|\Phi \varphi-\varphi^{0}\right\| \\
& =\max \left\{\sup _{t \in \mathbb{T}}\left|\int_{-\infty}^{t} e_{-\delta_{1}}(t, \sigma(s)) \varphi_{2}(s) \Delta s\right|\right. \\
& \quad \sup _{t \in \mathbb{T}} \mid \int_{-\infty}^{t} e_{-\delta_{2}}(t, \sigma(s)) \times\left[\beta(s) \varphi_{1}(s)-b(s) \varphi_{1}^{m}\right. \\
& \times \max \left\{\sup _{t \in \mathbb{T}} \int_{-\infty}^{t} e_{-\delta_{1}}(t, \sigma(s)) \Delta s\|\varphi\|,\right. \\
& \leq \max \left\{\frac{1}{\delta_{1}^{-}}, \frac{\beta^{+}+b^{+}}{\delta_{2}^{-}}\right\}\|\varphi\| \\
& \left.\quad=\theta\|\| \leq \frac{\theta L}{1-\theta} ; e_{-\infty}^{t}(t, \sigma(s)) \Delta s \times\left(\beta^{+}\|\varphi\|+b^{+}\|\varphi\|^{m}\right)\right\}
\end{aligned}
$$

that is, $\Phi \varphi \in \mathbb{X}_{0}$. So the mapping $\Phi$ is a self-mapping from $\mathbb{X}_{0}$ to $\mathbb{X}_{0}$. Next, we prove that the mapping $\Phi$ is a contraction mapping of $\mathbb{X}_{0}$. In fact, for any $\varphi=\left(\varphi_{1}, \varphi_{2}\right)^{T}, \psi=\left(\psi_{1}, \psi_{2}\right)^{T} \in$ $\mathbb{X}_{0}$, we can get

$$
\begin{aligned}
& \|\Phi \varphi-\Phi \psi\|_{B} \\
& =\max _{1 \leq i \leq 2}\left\{\sup _{t \in \mathbb{T}}\left|\Phi \varphi_{i}(t)-\Phi \psi_{i}(t)\right|\right\} \\
& \leq \max \left\{\sup _{t \in \mathbb{T}} \int_{-\infty}^{t} e_{-\delta_{1}}(t, \sigma(s))\left|\varphi_{2}(s)-\psi_{2}(s)\right| \Delta s,\right. \\
& \sup _{t \in \mathbb{T}} \int_{-\infty}^{t} e_{-\delta_{2}}(t, \sigma(s)) \\
& \times \mid \beta(s)\left(\varphi_{1}(s)-\psi_{1}(s)\right)-b(s) \\
& \times\left(\varphi_{1}^{m}(s-\tau(s))\right. \\
& \left.\left.-\psi_{1}^{m}(s-\tau(s))\right) \mid \Delta s\right\} \\
& \leq \max \left\{\frac{1}{\delta_{1}^{-}}\|\varphi-\psi\|,\right. \\
& \sup _{t \in \mathbb{\mathbb { T }}} \int_{-\infty}^{t} e_{-\delta_{2}}(t, \sigma(s)) \\
& \times \mid \beta(s)\left(\varphi_{1}(s)-\psi_{1}(s)\right) \\
& -b(s) m\left(\psi_{1}(s-\tau(s))+h(s)\right. \\
& \times\left(\varphi_{1}(s-\tau(s))\right. \\
& \left.\left.-\psi_{1}(s-\tau(s))\right)\right)^{m-1} \\
& \left.\times\left(\varphi_{1}(s-\tau(s))-\psi_{1}(s-\tau(s))\right) \mid \Delta s\right\}
\end{aligned}
$$




$$
\begin{aligned}
& =\max \left\{\frac{1}{\delta_{1}^{-}}\|\varphi-\psi\|,\right. \\
& \sup _{t \in \mathbb{T}} \int_{-\infty}^{t} e_{-\delta_{2}}(t, \sigma(s)) \\
& \quad \times \mid \beta(s)\left(\varphi_{1}(s)-\psi_{1}(s)\right) \\
& \quad-b(s) m\left((1-h(s)) \psi_{1}(s-\tau(s))\right. \\
& \times\left(\varphi_{1}(s-\tau(s))\right. \\
& \left.\quad-h(s) \varphi_{1}(s-\tau(s))\right)^{m-1}
\end{aligned}
$$

where $0 \leq h(s) \leq 1$. Noticing that $\gamma<1$, it is clear that the mapping $\Phi$ is a contraction mapping of $\mathbb{X}_{0}$. By the fixed point theorem of Banach space, $\Phi$ has a unique fixed point in $\mathbb{X}_{0}$. That is, (10) has a unique almost periodic solution in $\mathbb{X}_{0}$. This completes the proof of Theorem 18.

Theorem 19. Let $\left(H_{1}\right)-\left(H_{3}\right)$ hold. Then the almost periodic solution of (10) is globally exponentially stable.

Proof. By Theorem 18, (10) has an almost periodic solution $z_{*}(t)=\left(x_{*}(t), y_{*}(t)\right)^{T} \in \mathbb{X}_{0}$ with the initial value $\varphi_{*}(s)=$ $\left(\varphi_{* 1}(s), \varphi_{* 2}(s)\right)^{T}$. Suppose that $z(t)=(x(t), y(t))^{T}$ is an arbitrary solution of (10) with the initial value $\varphi(s)=$ $\left(\varphi_{1}(s), \varphi_{2}(s)\right)^{T}$. Denote $w(t)=(u(t), v(t))^{T}$, where $u(t)=$ $x(t)-x_{*}(t), v(t)=y(t)-y_{*}(t)$. Then it follows from (10) that

$$
\begin{gathered}
u^{\Delta}(t)=-\delta_{1}(t) u(t)+v(t), \\
v^{\Delta}(t)=-\delta_{2}(t) v(t)+\beta(t) u(t) \\
-b(t)\left(x^{m}(t-\tau(t))-x_{*}^{m}(t-\tau(t))\right) .
\end{gathered}
$$

The initial condition of (31) is

$$
\phi_{i}(s)=\varphi_{i}(s)-\varphi_{* i}(s), \quad s \in\left[-\tau^{+}, 0\right]_{\mathbb{T}}, \quad i=1,2 .
$$

Multiply both sides of the first equation of (31) by $e_{-\delta_{1}}(t, \sigma(s))$ and the second equation by $e_{-\delta_{2}}(t, \sigma(s))$, respectively. Then integrating on $\left[t_{0}, t\right]_{\mathbb{T}}$, where $t_{0} \in\left[-\tau^{+}, 0\right]_{\mathbb{T}}$, we get

$$
\begin{aligned}
u(t) & =\phi_{1}\left(t_{0}\right) e_{-\delta_{1}}\left(t, t_{0}\right)+\int_{t_{0}}^{t} e_{-\delta_{1}}(t, \sigma(s)) v(s) \Delta s, \\
v(t)= & \phi_{2}\left(t_{0}\right) e_{-\delta_{2}}\left(t, t_{0}\right) \\
& +\int_{t_{0}}^{t} e_{-\delta_{2}}(t, \sigma(s)) \\
& \times\left(\beta(s) u(s)-b(s)\left(x^{m}(s-\tau(s))\right.\right. \\
& \left.-x_{*}^{m}(s-\tau(s))\right) \Delta s .
\end{aligned}
$$

For positive a constant $\alpha$ with $-\alpha \in \mathscr{R}^{+}$, we have $e_{\ominus \alpha}\left(t, t_{0}\right)>1$, where $t \in\left[-\theta, t_{0}\right]_{\mathbb{T}}$. Take $M>1 /(1-\gamma)$; then it is obvious that $M>1$ and

$$
\begin{array}{r}
\left|z(t)-z_{*}(t)\right|_{1}=|\phi(t)|_{1} \leq\|\phi\| \leq M e_{\ominus \alpha}\left(t, t_{0}\right)\|\phi\|, \\
\forall t \in\left[-\tau^{+}, t_{0}\right]_{\mathbb{T}} .
\end{array}
$$

We claim that

$$
\left|z(t)-z_{*}(t)\right|_{1} \leq M e_{\ominus \alpha}\left(t, t_{0}\right)\|\phi\|, \quad \forall t \in\left(t_{0},+\infty\right)_{\mathbb{T}} .
$$

To prove this claim, we show that for any constant $p>1$, the following inequality holds:

$$
\left|z(t)-z_{*}(t)\right|_{1}<p M e_{\ominus \alpha}\left(t, t_{0}\right)\|\phi\|, \quad \forall t \in\left(t_{0},+\infty\right)_{\mathbb{T}},
$$

which implies that

$$
\begin{array}{r}
|u(t)|=\left|x(t)-x_{*}(t)\right|<p M e_{\ominus \alpha}\left(t, t_{0}\right)\|\phi\|, \\
\forall t \in\left(t_{0},+\infty\right)_{\mathbb{T}}, \\
|v(t)|=\left|y(t)-y_{*}(t)\right|<p M e_{\ominus \alpha}\left(t, t_{0}\right)\|\phi\|, \\
\forall t \in\left(t_{0},+\infty\right)_{\mathbb{V}} .
\end{array}
$$

By way of contradiction, assume that (36) does not hold. We will have the following three cases.

Case 1. Inequality (38) is true and (37) is not true. Then there exist $t_{1} \in\left(t_{0},+\infty\right)_{\mathbb{T}}$ and $\Theta \geq 1$ such that

$$
\begin{gathered}
\left|u\left(t_{1}\right)\right|=\Theta p M e_{\ominus \alpha}\left(t_{1}, t_{0}\right)\|\phi\|, \\
|u(t)|<\Theta p M e_{\ominus \alpha}\left(t, t_{0}\right)\|\phi\|, \quad t \in\left(t_{0}, t_{1}\right)_{\mathbb{T}} .
\end{gathered}
$$


Note that, in view of (33), we have

$$
\begin{aligned}
& \left|u\left(t_{1}\right)\right|=\left|\phi_{1}\left(t_{0}\right) e_{-\delta_{1}}\left(t_{1}, t_{0}\right)+\int_{t_{0}}^{t_{1}} e_{-\delta_{1}}\left(t_{1}, \sigma(s)\right) v(s) \Delta s\right| \\
& \leq e_{-\delta_{1}^{-}}\left(t_{1}, t_{0}\right)\|\phi\| \\
& +\int_{t_{0}}^{t_{1}} e_{-\delta_{1}^{-}}\left(t_{1}, \sigma(s)\right) \Theta p M e_{\ominus \alpha}\left(s, t_{0}\right)\|\phi\| \Delta s \\
& \leq e_{-\delta_{1}^{-}}\left(t_{1}, t_{0}\right) e_{\ominus(-\alpha)}\left(t_{1}, t_{0}\right)\|\phi\| \\
& +\Theta p M e_{\ominus \alpha}\left(t_{1}, t_{0}\right)\|\phi\| \\
& \times \int_{t_{0}}^{t_{1}} e_{-\delta_{1}^{-}}\left(t_{1}, \sigma(s)\right) e_{\ominus \alpha}\left(s, t_{1}\right) \Delta s \\
& \leq e_{\ominus \alpha}\left(t_{1}, t_{0}\right)\|\phi\|+\Theta p M e_{\ominus \alpha}\left(t_{1}, t_{0}\right)\|\phi\| \\
& \times \int_{t_{0}}^{t_{1}} e_{-\delta_{1}^{-}}\left(t_{1}, \sigma(s)\right) \Delta s \\
& =e_{\ominus \alpha}\left(t_{1}, t_{0}\right)\|\phi\|+\frac{1}{-\delta_{1}^{-}} \Theta p M e_{\ominus \alpha}\left(t_{1}, t_{0}\right)\|\phi\| \\
& \times \int_{t_{0}}^{t_{1}} \delta_{1}^{-} e_{-\delta_{1}}\left(t_{1}, \sigma(s)\right) \Delta s \\
& =\Theta p M e_{\ominus \alpha}\left(t_{1}, t_{0}\right)\|\phi\| \\
& \times\left(\frac{1}{\Theta p M}-\frac{1}{\delta_{1}^{-}}\left(e_{-\delta_{1}^{-}}\left(t_{1}, t_{0}\right)-1\right)\right) \\
& <\Theta p M e_{\ominus \alpha}\left(t_{1}, t_{0}\right)\|\phi\|\left(\frac{1}{\Theta M}+\frac{1}{\delta_{1}^{-}}\right) \\
& <\Theta p M e_{\ominus \alpha}\left(t_{1}, t_{0}\right)\|\phi\| \text {. }
\end{aligned}
$$

In the above proof we use the inequality $e_{-\delta_{1}^{-}}\left(t_{1}, t_{0}\right)<1$. Thus we get a contradiction.

Case 2. Inequality (37) is true and (38) is not true. Then there exist $t_{2} \in\left(t_{0},+\infty\right)_{\mathbb{T}}$ and $\Theta_{1} \geq 1$ such that

$$
\begin{gathered}
\left|v\left(t_{2}\right)\right|=\Theta_{1} p M e_{\ominus \alpha}\left(t_{2}, t_{0}\right)\|\phi\|, \\
|v(t)|<\Theta_{1} p M e_{\ominus \alpha}\left(t, t_{0}\right)\|\phi\|, \quad t \in\left(t_{0}, t_{2}\right)_{\mathbb{T}} .
\end{gathered}
$$

Note that, in view of (33), we have

$$
\begin{aligned}
& \left|v\left(t_{2}\right)\right| \\
& =\mid \phi_{2}\left(t_{0}\right) e_{-\delta_{2}}\left(t_{2}, t_{0}\right)+\int_{t_{0}}^{t_{2}} e_{-\delta_{2}}\left(t_{2}, \sigma(s)\right) \\
& \times(\beta(s) u(s)-b(s) \\
& \times\left(x^{m}(s-\tau(s))\right. \\
& \left.\left.-x_{*}^{m}(s-\tau(s))\right)\right) \Delta s \mid
\end{aligned}
$$

$$
\begin{aligned}
& \leq e_{-\delta_{2}^{-}}\left(t_{2}, t_{0}\right)\|\phi\| \\
& +\int_{t_{0}}^{t_{2}} e_{-\delta_{2}^{-}}\left(t_{2}, \sigma(s)\right) \mid \beta(s) u(s)-b(s) \\
& \times\left(x^{m}(s-\tau(s))\right. \\
& \left.-x_{*}^{m}(s-\tau(s))\right) \mid \Delta s \\
& =e_{-\delta_{2}^{-}}\left(t_{2}, t_{0}\right)\|\phi\| \\
& +\int_{t_{0}}^{t_{2}} e_{-\delta_{2}^{-}}\left(t_{2}, \sigma(s)\right) \\
& \times \mid \beta(s) u(s)-b(s) m\left(x_{*}(s-\tau(s))+\zeta(s)\right. \\
& \times(x(s-\tau(s)) \\
& \left.\left.-x_{*}(s-\tau(s))\right)\right)^{m-1} \\
& \times\left(x(s-\tau(s))-x_{*}(s-\tau(s))\right) \mid \Delta s \\
& =e_{-\delta_{2}^{-}}\left(t_{2}, t_{0}\right)\|\phi\| \\
& +\int_{t_{0}}^{t_{2}} e_{-\delta_{2}^{-}}\left(t_{2}, \sigma(s)\right) \\
& \times \mid \beta(s) u(s)-b(s) m\left((1-\zeta(s)) x_{*}(s-\tau(s))\right. \\
& +\zeta(s) x(s-\tau(s)))^{m-1} \\
& \times\left(x(s-\tau(s))-x_{*}(s-\tau(s))\right) \mid \Delta s \\
& \leq e_{-\delta_{2}^{-}}\left(t_{2}, t_{0}\right)\|\phi\| \\
& +\int_{t_{0}}^{t_{2}} e_{-\delta_{2}^{-}}\left(t_{2}, \sigma(s)\right)\left[\beta^{+}|u(s)|+m b^{+}\right. \\
& \times \mid\left((1-\zeta(s)) x_{*}(s-\tau(s))\right. \\
& +\zeta(s) x(s-\tau(s)))^{m-1} \\
& \times(x(s-\tau(s)) \\
& \left.\left.-x_{*}(s-\tau(s))\right) \mid\right] \Delta s
\end{aligned}
$$$$
\leq e_{-\delta_{2}^{-}}\left(t_{2}, t_{0}\right)\|\phi\|
$$$$
+\int_{t_{0}}^{t_{2}} e_{-\delta_{2}^{-}}\left(t_{2}, \sigma(s)\right)
$$$$
\times\left[\beta^{+}|u(s)|+m b^{+}\left(\frac{2 L}{1-\theta}\right)^{m-1}|u(s-\tau(s))|\right] \Delta s
$$$$
\leq e_{-\delta_{2}^{-}}\left(t_{2}, t_{0}\right)\|\phi\|+\beta^{+} \Theta_{1} p M e_{\ominus \alpha}\left(t_{2}, t_{0}\right)\|\phi\|
$$$$
\times \int_{t_{0}}^{t_{2}} e_{-\delta_{2}^{-}}\left(t_{2}, \sigma(s)\right) \Delta s
$$$$
+m b^{+}\left(\frac{2 L}{1-\theta}\right)^{m-1} \Theta_{1} p M e_{\ominus \alpha}\left(t_{2}, t_{0}\right)\|\phi\|
$$$$
\times \int_{t_{0}}^{t_{2}} e_{-\delta_{2}^{-}}\left(t_{2}, \sigma(s)\right) e_{\ominus \alpha}\left(s-\tau(s), t_{2}\right) \Delta s
$$ 


$$
\begin{aligned}
& \leq e_{-\delta_{2}^{-}}\left(t_{2}, t_{0}\right) e_{\ominus \alpha}\left(t_{2}, t_{0}\right)\|\phi\| \\
& +\frac{1}{-\delta_{2}^{-}} \beta^{+} \Theta_{1} p M e_{\ominus \alpha}\left(t_{2}, t_{0}\right)\|\phi\| \\
& \times \int_{t_{0}}^{t_{2}}\left(-\delta_{2}^{-}\right) e_{-\delta_{2}^{-}}\left(t_{2}, \sigma(s)\right) \Delta s \\
& +\frac{1}{-\delta_{2}^{-}} m b^{+}\left(\frac{2 L}{1-\theta}\right)^{m-1} \Theta_{1} p M e_{\ominus \alpha}\left(t_{2}, t_{0}\right) \\
& \times\|\phi\| \exp \left\{-\alpha \tau^{-}\right\} \int_{t_{0}}^{t_{2}}\left(-\delta_{2}^{-}\right) e_{-\delta_{2}^{-}}\left(t_{2}, \sigma(s)\right) \Delta s \\
& \leq e_{\ominus \alpha}\left(t_{2}, t_{0}\right)\|\phi\|-\frac{1}{\delta_{2}^{-}} \beta^{+} \Theta_{1} p M e_{\ominus \alpha}\left(t_{2}, t_{0}\right) \\
& \times\|\phi\|\left(e_{-\delta_{2}^{-}}\left(t_{2}, t_{0}\right)-1\right) \\
& -\frac{1}{\delta_{2}^{-}} m b^{+}\left(\frac{2 L}{1-\theta}\right)^{m-1} \Theta_{1} p M e_{\ominus \alpha}\left(t_{2}, t_{0}\right) \\
& \times\|\phi\| \exp \left\{-\alpha \tau^{-}\right\}\left(e_{-\delta_{2}^{-}}\left(t_{2}, t_{0}\right)-1\right) \\
& \leq \Theta_{1} p M e_{\ominus \alpha}\left(t_{2}, t_{0}\right)\|\phi\| \\
& \times\left(\frac{1}{\Theta_{1} p M}+\frac{\beta^{+}+m b^{+}(2 L /(1-\theta))^{m-1}}{\delta_{2}^{-}}\right) \\
& <\Theta_{1} p M e_{\ominus \alpha}\left(t_{2}, t_{0}\right)\|\phi\| \\
& \times\left(\frac{1}{\Theta_{1} M}+\frac{\beta^{+}+m b^{+}(2 L /(1-\theta))^{m-1}}{\delta_{2}^{-}}\right) \\
& <\Theta_{1} p M e_{\ominus \alpha}\left(t_{2}, t_{0}\right)\|\phi\|,
\end{aligned}
$$

where $0 \leq \zeta(s) \leq 1$. We also get a contradiction.

Case 3. Inequalities (37) and (38) are both untrue. By Case 1 and Case 2, we can obtain a contradiction. Therefore, (36) holds. Let $p \rightarrow 1$; then (35) holds. We can take $-\lambda=\ominus \alpha$; then $\lambda>0$ and $-\lambda \in \mathscr{R}^{+}$. Hence, we have that

$$
\begin{array}{r}
\left|z(t)-z_{*}(t)\right|_{1} \leq M\left\|\varphi-\varphi_{*}\right\| e_{-\lambda}\left(t, t_{0}\right), \\
t \in\left[-\tau^{+}, \infty\right)_{\mathbb{T}}, \quad t \geq t_{0},
\end{array}
$$

which implies that the periodic solution $z_{*}(t)$ of $(10)$ is globally exponentially stable. This completes the proof of Theorem 19.

Remark 20. It is easy to see that when $\mathbb{T}=\mathbb{R}$, Theorem 18 extends the results obtained in [5-8]. Moreover, when $\mathbb{T}=\mathbb{R}$, $c(t) \equiv 0, a(t) \equiv a$, and $b(t) \equiv b$, Theorem 18 improves the results obtained in [6].

\section{Numerical Examples}

In this section, we present numerical examples to illustrate the feasibility of our results obtained in Section 3.
Example 1. Consider the delay Duffing equation on an almost periodic time scale $\mathbb{T}$ :

$$
\begin{aligned}
& \left(x^{\Delta}\right)^{\Delta}(t)+(3.1+0.01 \sin \sqrt{2} t) x^{\Delta}(t)+(2+0.01 \cos t) x(t) \\
& \quad+(0.03+0.02 \cos \sqrt{2} t) x^{3}(t-\cos \sqrt{2} t) \\
& =0.03-0.01 \sin \sqrt{3} t .
\end{aligned}
$$

It is easy to see that $c(t)=3.1+0.01 \sin \sqrt{2} t, a(t)=$ $-2-0.01 \cos t, b(t)=0.03+0.02 \cos \sqrt{2} t, p(t)=0.03-$ $0.01 \sin \sqrt{3} t, \tau(t)=\cos \sqrt{2} t$, and $m=3$. If we set $\delta_{1}(t)=1.6+$ $0.01 \sin t$, then we have $\delta_{2}(t)=1.5+0.01 \sin t-0.01 \sin (\sigma(t))$, $\beta(t)=-2-0.01 \cos t+0.01(\sin t)^{\Delta}+(1.6+0.01 \sin t)(1.5+$ $0.01 \sin t-0.01 \sin (\sigma(t)))$. If $\mathbb{T}=\mathbb{R}$, then $\mu(t)=0$, and if $\mathbb{T}=\mathbb{Z}$, then $\mu(t)=1$. We can verify for the above two cases; $\theta \approx 0.43<1, \gamma \approx 0.4<1, L /(1-\theta) \approx 0.081<1$. That is, all conditions of Theorem 18 are satisfied. Hence, (44) has an almost periodic solution, which is globally exponentially stable.

Example 2. Consider the delay Duffing equation on $\mathbb{R}$ :

$$
\begin{aligned}
x^{\prime \prime}(t) & +(2+0.01 \cos \sqrt{3} t) x^{\prime}(t)+(1+0.01 \sin t) x(t) \\
& +(0.04+0.03 \cos \sqrt{2} t) x^{2}(t-\sin \sqrt{3} t) \\
= & 0.05-0.03 \cos \sqrt{2} t .
\end{aligned}
$$

It is easy to see that $c(t)=2+0.02 \cos \sqrt{3} t, a(t)=$ $-2-0.01 \sin t, b(t)=0.04+0.03 \cos \sqrt{2} t, p(t)=0.05-$ $0.03 \cos \sqrt{2} t, \tau(t)=\sin \sqrt{3} t, m=2, \sigma(t)=t$, and $\mu(t)=0$. If we set $\delta_{1}(t)=1+0.02 \cos \sqrt{3} t$, then we have $\delta_{2}(t)=1, \beta(t)=$ $0.04 \cos \sqrt{3} t-0.01 \sin t$. Hence, we have that $\theta \approx 0.57<1$, $\gamma \approx 0.39<1$, and $L /(1-\theta) \approx 0.261<1$, which imply that all conditions of Theorem 18 are satisfied. Hence, (45) has an almost periodic solution, which is globally exponentially stable.

\section{Conclusion}

Using the exponential dichotomy of linear dynamic equations on time scales and the time scale calculus theory, some sufficient conditions are derived to guarantee the existence and exponential stability of almost periodic solutions for a class of Duffing equation on time scales. To the best of our knowledge, the results presented here have not appeared in the related literature. Besides, the results obtained in this paper possess feasibility. Moreover, the method in this paper may be applied to some other differential equations on time scales.

\section{Conflict of Interests}

The authors declare that there is no conflict of interests regarding the publication of this paper. 


\section{Acknowledgments}

The authors thank the referees for their careful reading of the paper, support, and insightful comments. This work is supported by the National Natural Sciences Foundation of People's Republic of China under Grant 11361072.

\section{References}

[1] D. Hao and S. Ma, "Semilinear Duffing equations crossing resonance points," Journal of Differential Equations, vol. 133, no. 1, pp. 98-116, 1997.

[2] B. Liu, "Boundedness of solutions for semilinear Duffing equations," Journal of Differential Equations, vol. 145, no. 1, pp. 119144, 1998.

[3] X. P. Liu, M. Jia, and R. Ren, "Existence and uniqueness of periodic solutions to a type of Duffing equation with complex deviating argument," Acta Mathematica Scientia A, vol. 27, no. 1, pp. 37-44, 2007 (Chinese).

[4] Z. Wang, L. Qian, S. Lu, and J. Cao, "The existence and uniqueness of periodic solutions for a kind of Duffing-type equation with two deviating arguments," Nonlinear Analysis: Theory, Methods \& Applications, vol. 73, no. 9, pp. 3034-3043, 2010.

[5] W. Zeng, "Almost periodic solutions for nonlinear Duffing equations," Acta Mathematica Sinica, vol. 13, no. 3, pp. 373-380, 1997.

[6] Q. Zhou and B. Liu, "New results on almost periodic solutions for a class of nonlinear Duffing equations with a deviating argument," Applied Mathematics Letters, vol. 22, no. 1, pp. 6-11, 2009.

[7] L. Q. Peng and W. T. Wang, "Positive almost periodic solutions for a class of nonlinear Duffing equations with a deviating argument," Electronic Journal of Qualitative Theory of Differential Equations, vol. 6, pp. 1-12, 2010.

[8] Y. Xu, "Existence and uniqueness of almost periodic solutions for a class of nonlinear Duffing system with time-varying delays," Electronic Journal of Qualitative Theory of Differential Equations, vol. 80, pp. 1-9, 2012.

[9] M. Bohner and A. Peterson, Dynamic Equations on Time Scales: An Introduction with Applications, Birkhäuser, Boston, Mass, USA, 2001.

[10] Y. Li and C. Wang, "Uniformly almost periodic functions and almost periodic solutions to dynamic equations on time scales," Abstract and Applied Analysis, vol. 2011, Article ID 341520, 22 pages, 2011.

[11] J. Zhang, M. Fan, and H. Zhu, "Existence and roughness of exponential dichotomies of linear dynamic equations on time scales," Computers \& Mathematics with Applications, vol. 59, no. 8, pp. 2658-2675, 2010. 


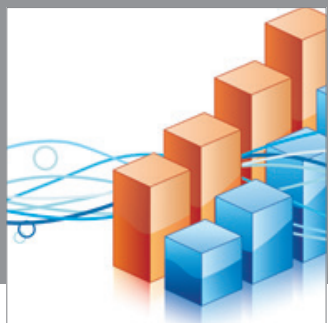

Advances in

Operations Research

mansans

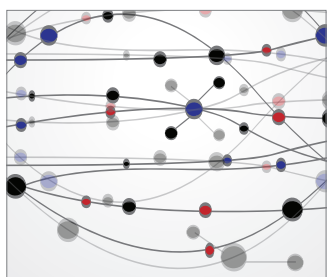

The Scientific World Journal
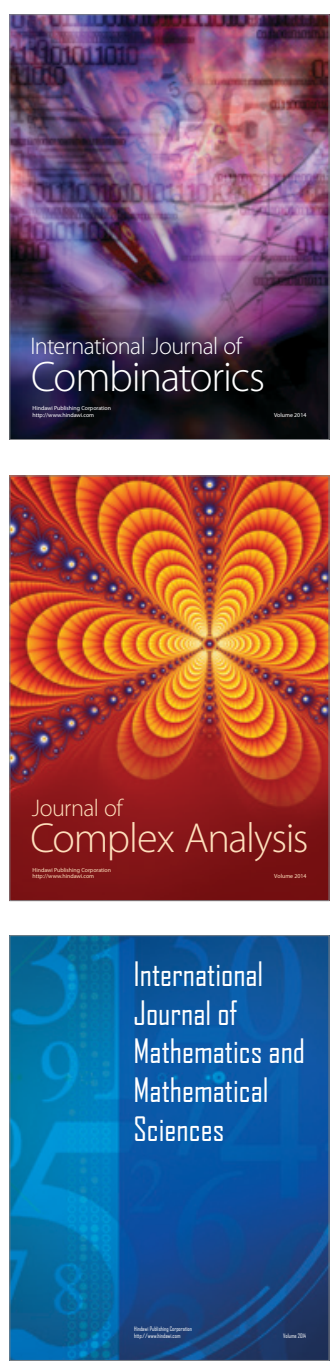
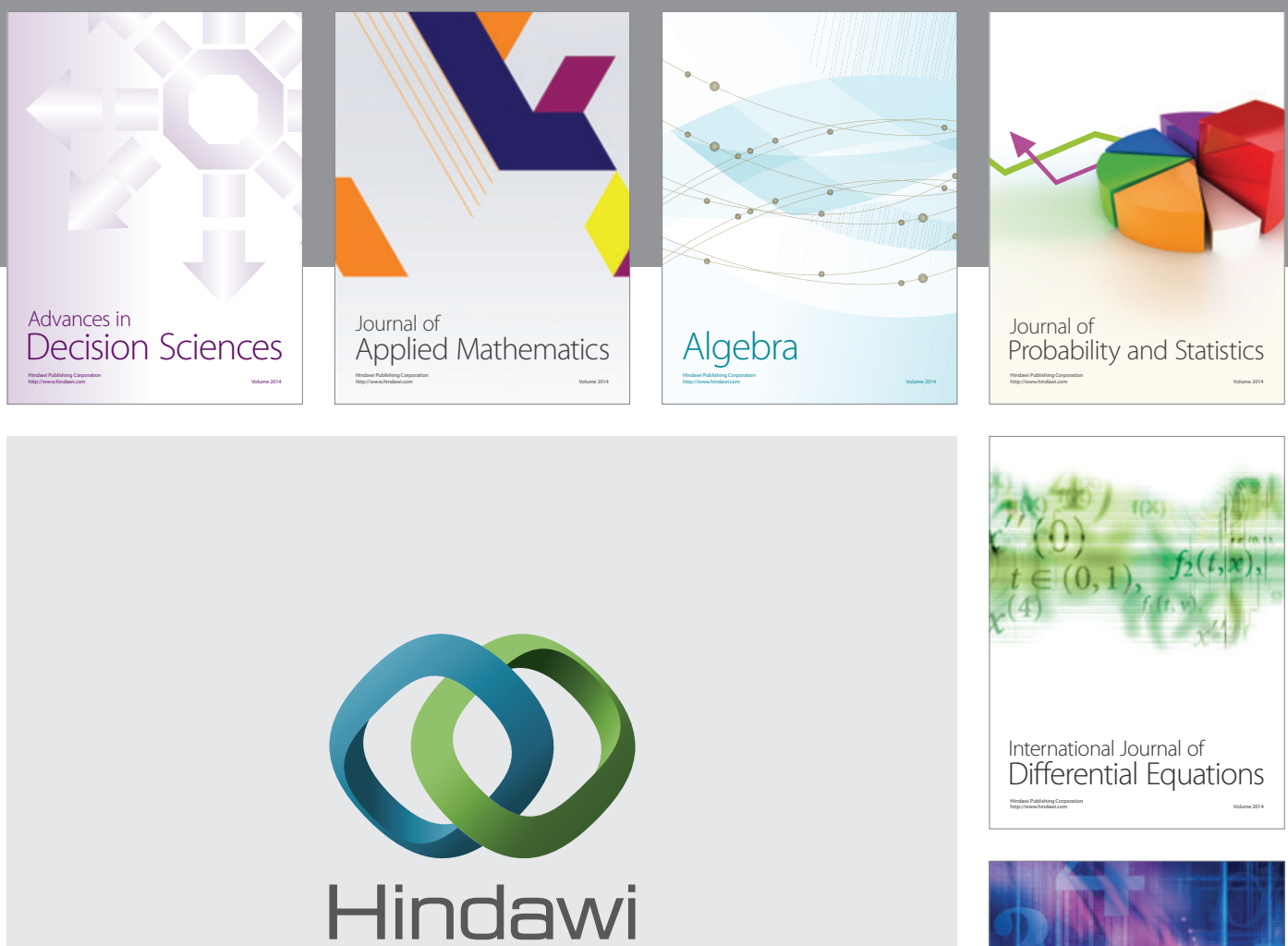

Submit your manuscripts at http://www.hindawi.com
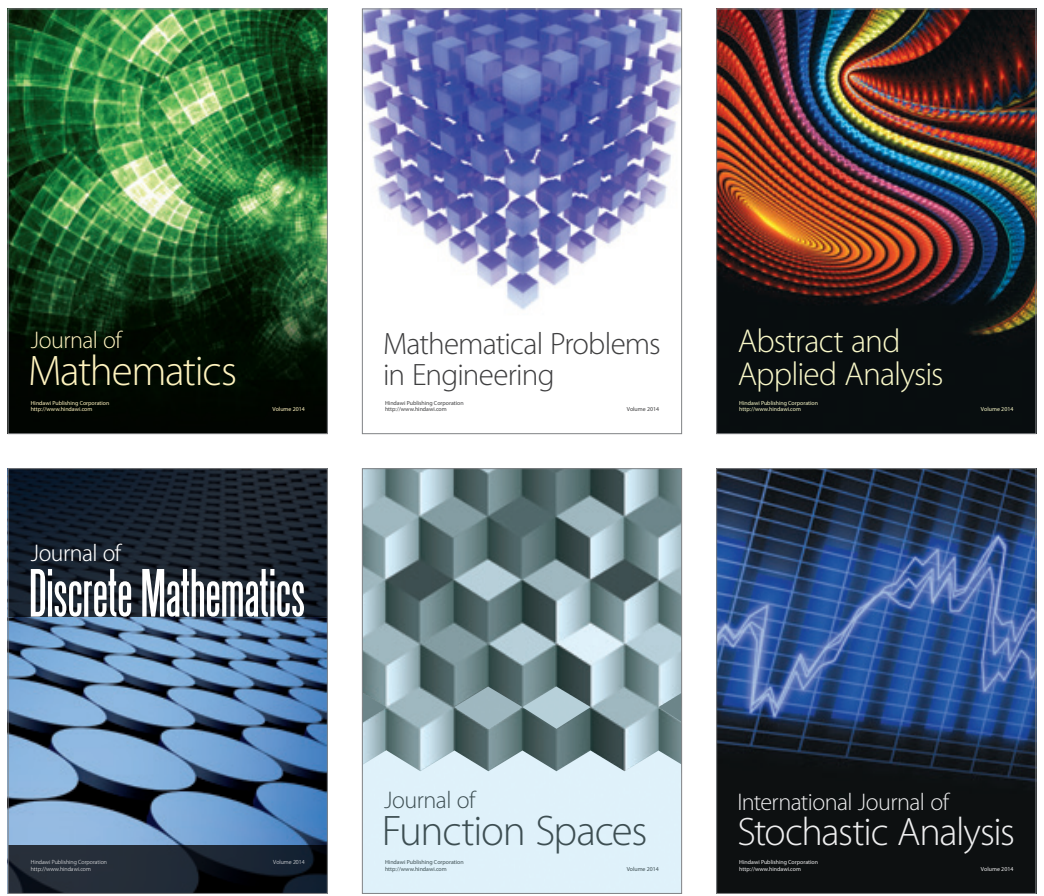

Journal of

Function Spaces

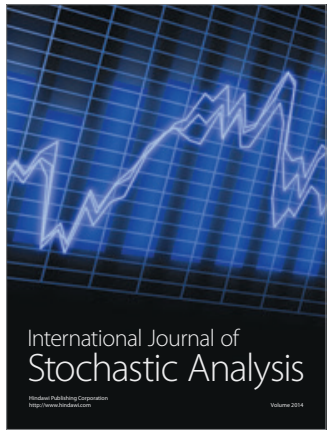

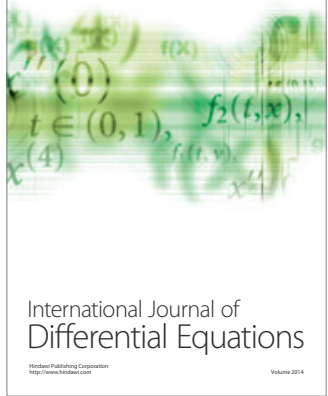
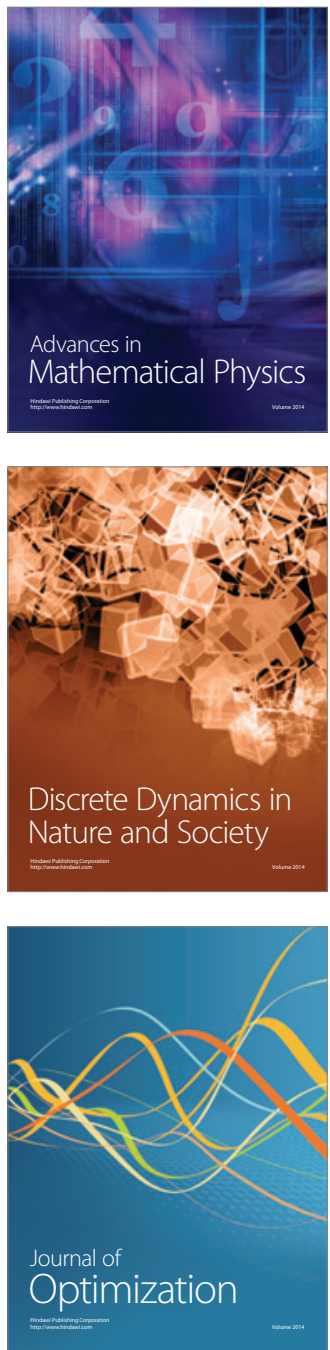